\title{
Upscaling nanoparticle synthesis by sputter deposition in ionic liquids
}

\author{
M. Meischein • A. Ludwig
}

Received: 11 March 2021 / Accepted: 25 May 2021 / Published online: 12 June 2021

(C) The Author(s) 2021

\begin{abstract}
Upscaling of nanoparticle fabrication by sputtering into an ionic liquid is shown for the example of $\mathrm{Cu}$. Long-time sputtering ( $24 \mathrm{~h}$ ) into a large amount $(50 \mathrm{~mL})$ of the ionic liquid 1-butyl3-methylimidazolium bis-(trifluoromethylsulfonyl) imide $[\mathrm{Bmim}]\left[(\mathrm{Tf})_{2} \mathrm{~N}\right]$ yields an amount of approximately $1 \mathrm{~g} \mathrm{Cu}$ nanoparticles (mean spherical diameter $(2.6 \pm 1.1) \mathrm{nm})$, stabilized in ionic liquid without agglomerations. Extraction of $\mathrm{Cu}$ nanoparticles from the stabilizing ionic liquid was performed with the capping agent hexadecylamine. Extracted particles could be redispersed in other solvents, thus enabling applications of sputtered nanoparticles beyond ionic liquids.
\end{abstract}

Keywords Ionic liquids · Sputter deposition · Copper nanoparticles · Upscaling nanoparticle synthesis

\section{Introduction}

Metallic nanoparticles (NPs) have many application possibilities, e.g., in medicine (Breisch et al. 2019; Conde et al. 2012; Weissleder et al. 1990; Ghotekar

\section{Meischein · A. Ludwig $(\bowtie)$}

Materials Discovery and Interfaces, Institute for Materials, Faculty of Mechanical Engineering, Ruhr University Bochum, Universitätsstr. 150, 44780 Bochum, Germany e-mail: alfred.ludwig@rub.de et al. 2021; Javed et al. 2021), optics (Li and Zhang 2009; Chen et al. 2021; Khurana and Jaggi 2021), electronics (Karmakar et al. 2011; Sudhan et al. 2021), and catalysis (Migowski and Dupont 2007; Kharisov et al. 2019; Astruc 2008; Al-Madanat et al. 2021). For applications, an effective large-scale synthesis process of nanoparticles is necessary. NPs are most frequently prepared by wet chemical approaches comprising the reduction or decomposition of metal precursors (Bracey et al. 2009; Garzón-Manjón et al. 2018a, 2015; Itoh et al. 2004; Raut et al. 2009; Sahoo et al. 2009; Yang et al. 2009; Okazoe et al. 2020), but also, mechanical techniques like milling or attrition (Datta et al. 2000; Baraton 2003; Liu et al. 2020) can be used, representing bottom-up and top-down methods for NP synthesis. In literature, several publications exist concerning NP synthesis enabling an upscaling of the NP yield to the gram level with techniques different than sputtering on ILs. Besides mechanical processes like ball milling (Lam et al. 2000), chemical or thermal decomposition of precursors (Cui et al. 2009; Kim et al. 2015; Lee et al. 2005; Song and Chen 2003; Wen et al. 2012; Lee et al. 2008; Lee and Chen 2006) and (flame) spray synthesis (Athanassiou et al. 2006; Wang et al. 2020) are commonly used for a high yield of NP amounts. Upscaling synthesis of NPs using ionic liquids (ILs) was also reported (Lu et al. 2009; Zheng et al. 2009; Xiao et al. 2013).

The unique physical and chemical characteristics of ILs make them an interesting medium for chemical 
and industrial processes, for example, in catalysis (Luska et al. 2015) and as reaction and scavenging media (Plechkova and Seddon 2008). ILs consist only of specially structured cations and anions. They can be regarded as liquid salts at temperature $<100{ }^{\circ} \mathrm{C}$. Taking all suitable cations and anions for IL synthesis into account, $10^{18}$ ILs could be fabricated (Holbrey and Seddon 1999), serving as a vast pool for different applications and task-specific design of individual ILs. In general, ILs possess outstanding characteristics making them versatile media for NP synthesis. Their negligible vapor pressure (Wasserscheid and Keim 2000; Kuwabata et al. 2014) provides the application of ILs as green chemistry solvents and clean substrates for vacuum processes (Vekariya 2017).

Wet chemical NP synthesis routes can be applied in ILs and comprise a huge part of the literature dealing with large-scale NP synthesis. One drawback of those reactions are chemical byproducts which can be present in the resulting colloidal solution, which can impede applications of the NPs (Torimoto et al. 2006).

Overcoming these drawbacks by avoiding precursor chemicals and their decomposition products can be achieved by physical vapor deposition (PVD) of metals in ILs (Richter et al. 2010, 2011). Thermal evaporation for NP synthesis in ILs has been applied (Richter et al. 2011), but shows the drawback of necessary high thermal energies depending on the evaporation temperatures of the used elements. Additionally, for fabrication of binary NPs, the different specific vapor pressures of the elements make the adjustment of a desired alloy composition challenging (König et al. 2014). Sputter deposition of metals into pure ILs is a PVD technique for fabrication of custom- and task-designed NPs with adjustable size and morphology, controlled by the used IL, and multinary composition without byproducts or organic impurities (Torimoto et al. 2006; Hatakeyama et al. 2009). Typical NP diameters obtained from sputtering into ILs range from 1 to $10 \mathrm{~nm}$ (König et al. 2014; Meischein et al. 2019; Dupont and Scholten 2010; Nguyen and Yonezawa 2018; Meyer et al. 2018). Depending on the elemental composition of the NPs, the size distributions can show different ranges. For example, $\mathrm{Au}$ NPs show typically diameters far below $10 \mathrm{~nm}, \mathrm{Cu}$ NPs sometimes reach diameters of and above $10 \mathrm{~nm}$, and Ag NPs are likely bigger than $10 \mathrm{~nm}$ up to diameters of $15 \mathrm{~nm}$ but rarely more than $20 \mathrm{~nm}$. However, the NP size distribution could be expanded towards NPs with diameters above $50 \mathrm{~nm}$ by sputtering onto IL mixtures (Meischein et al. 2020). The NP formation occurs without any stabilization agents and the composition of binary or multinary NPs can be determined by co-sputtering and the specific sputter rates of the elements controlled by the applied sputtering powers (König et al. 2014; Hirano et al. 2013). The (multinary) NP formation by sputtering into ILs is not limited to reaction mechanisms and the availability of precursor chemicals since all vacuum compatible chemical elements can be used as target materials for (co-)sputtering (König et al. 2014; Meischein et al. 2019; Meyer et al. 2018).

In the literature, there are reports which applied sputtering for a potential upscaled synthesis of NPs. For example, sputtering of $\mathrm{Au}$ on a powder substrate resulted in well-dispersed NPs, representing a synthesis technique showing scalability and no waste stream (Veith et al. 2005). Since the potential of sputtering for large-scale NP synthesis was indicated in literature and since sputter deposition in ILs is a powerful and versatile synthesis tool for NP production, we aimed to increase the obtained NP amount over the usual scientific scale (usually a few $\geq \mathrm{g}$ ) by an extended sputter deposition of $\mathrm{Cu}$ into the IL [Bmim] $\left[(\mathrm{Tf})_{2} \mathrm{~N}\right]$. We increased the IL amount and the deposition time and decreased the distance from the target to the receiving IL with respect to our usual standard sputter parameters (König et al. 2014; Meischein et al. 2019, 2020; Meyer et al. 2018; Garzón-Manjón et al. 2018b; Löffler et al. 2018) in order to increase the sputter rate. The challenges of this approach are (I) to not exceed the IL stabilization capability by deposition too much material into the IL reaching a concentration which could not be stabilized (i.e., deposition power and duration of the process need to be adapted) and (II) to not degrade the IL by too much heat from the deposition process (i.e., the target should be not too close to the IL and the sputter power not too high). With an optimized experimental setup, we show the appropriateness of sputtering into ILs as a large-scale NP synthesis method.

The capping agent hexadecylamine (HDA) is used for extracting the $\mathrm{Cu}$ NPs out of the stabilizing IL. By addition of the capping agent to the $\mathrm{Cu}$ NP suspension, which is commonly used as stabilization mechanism in NP synthesis methods (Meshesha et al. 2009), the NPs precipitate due to strong interactions of the 
HDA with $\mathrm{Cu}$ surrounding the NP surface and forming a surfactant cage around the individual NPs. The extremely low solubility of HDA in the IL, caused by the long apolar alkyl chain, leads to precipitation of the capped NPs out of the IL, ending as flocculated powder at the bottom of the reaction vessel. The HDA-capped $\mathrm{Cu}$ NPs could be dissolved back into non-polar solvents or used for further investigations (König et al. 2014). TEM investigations of the assputtered and HDA-capped, re-dissolved NPs prove the success of the upscaled synthesis process, resulting in well-distributed NPs in ILs. Inductively coupled plasma mass spectroscopy (ICP-MS) was used to determine the amount of metal in the extracted powder and in the sputtered IL to prove if a sufficient amount of material for upscaling can be stabilized as NPs in ILs.

\section{Methods and materials}

The sample synthesis process was started by sputtering of $\mathrm{Cu}$ into IL for the NP formation, followed by the $\mathrm{Cu}$ precipitation process using HDA as capping agent and the extraction of the precipitated $\mathrm{Cu}$ NPs from the NP/IL suspension.

Upscaled sputter synthesis of Cu NP in IL

As liquid "substrate" for the sputter deposition of the NPs, the IL 1-butyl-3-methylimidazolium bis(trifluoromethylsulfonyl)imide [Bmim] $\left[(\mathrm{Tf})_{2} \mathrm{~N}\right]$ was used. This IL was purchased from IoLiTec (Heilbronn, Germany) with purity $>99 \%$, halide content $<100 \mathrm{ppm}$, and water content $51 \mathrm{ppm}$. Without further purification, the IL was stored and processed under Ar atmosphere in a glovebox (water and oxygen content both $<0.5 \mathrm{ppm}$ ). A commercial co-sputter system (AJA POLARIS-5 from AJA International, Inc., North Scituate, MA, USA) with 1.5-in.-diameter magnetron sputter cathodes and multiple sputter source DC power supplies (DC-XS 1500 from AJA International, Inc., North Scituate, MA, USA) were used for all sputter depositions. The process gas was Ar (purity 99.9999\%, Praxair, Düsseldorf, Germany). A 38.1-mm-diameter $\times$ 3.175-mm-thick $\mathrm{Cu}$ target (purity 99.99\%, EvoChem, Offenbach am Main, Germany) was used.
For the upscaled deposition, $50 \mathrm{~mL}$ of the IL $[\mathrm{Bmim}]\left[(\mathrm{Tf})_{2} \mathrm{~N}\right]$ was filled into a clean petri dish (110 $\mathrm{mm}$ inner diameter $\times 20 \mathrm{~mm}$ height) inside the glovebox. Prior to the deposition, the IL was evacuated inside the sputter chamber for $96 \mathrm{~h}$ in order to remove remaining water and oxygen from the transport out of the glovebox into the sputter chamber. The chamber pressure was $1.60 * 10^{-4}$ $\mathrm{Pa}$ before the deposition. For plasma ignition, the chamber pressure was set to $1.33 \mathrm{~Pa}$. After plasma ignition, the target was pre-cleaned for $120 \mathrm{~s}$ at $20 \mathrm{~W}$ sputter power with a closed shutter in front of the target, a rotation of the substrate plate of 30 rotations per minute and a successive reduction of the Ar pressure to the deposition pressure of $0.5 \mathrm{~Pa}$.

Pre-cleaning of the target was performed for removing possible oxide layers (Meyer et al. 2018), followed by adjusting the deposition power to $30 \mathrm{~W}(405 \mathrm{~V}, 75 \mathrm{~mA})$ and opening the shutter in front of the cathode for the specific deposition time. To reach the total deposition time of $24 \mathrm{~h}$, the deposition was conducted over 3 days with $9 \mathrm{~h}, 9 \mathrm{~h}$, and $6 \mathrm{~h}$ sputtering time per day. Between the depositions, the sputter chamber was continuously pumped when the sputter gas line was closed after each deposition to achieve that the vacuum never exceeded the sputter pressure during the deposition processes. The cathode was tilted by an angle of $18^{\circ}$ (standard $12^{\circ}$ ) between the normal of the IL surface and the target normal. The distance between the IL surface and the cathode was decreased from $11 \mathrm{~cm}$ (standard) to $9 \mathrm{~cm}$ by placing another pair of petri dishes below the IL filled dish. A homogeneous deposition was achieved on the substrate due to rotation and cathode tilt. After the deposition, right after opening the chamber, $300=\mathrm{L}$ of the sputtered $\mathrm{Cu}$ NP/IL suspension was separated from the IL dish and transferred into the glovebox for storage and TEM sample preparation of the untreated IL. The remaining Cu IL volume was filled into a glass bottle and kept in air since for the capping procedure, acetonitrile was used for washing the IL and thus a transfer into the glovebox would not be possible.

Subsequent to the deposition in IL, the sputter rate was determined using the identical sputter system configuration and deposition parameters 
(except deposition time) as for the IL deposition. The rate was determined using $\mathrm{Si} / \mathrm{SiO}_{2}$ pieces photolithographically structured with a photoresist lift-off cross pattern for film thickness measurements, also placed on a closed pair of petri dishes. The deposition time was $30 \mathrm{~min}$ to guarantee a sufficient film thickness. After deposition, the pieces were stored for $4 \mathrm{~h}$ in technical acetone to remove the lift-off photoresist and cleaned afterwards with technical acetone (purity $\geq 99.5 \%$ ) and isopropanol (purity $\geq 99.7 \%$ ). The film thicknesses was measured with a profilometer on 27 measurement points revealing a $\mathrm{Cu}$ deposition rate of $0.143 \mathrm{~nm} / \mathrm{s}$ for this chamber configuration, which is approximately $80 \%$ higher than the usual $\mathrm{Cu}$ rate $(0.08 \mathrm{~nm} / \mathrm{s})$ used in our standard scientific chamber configuration (Meischein et al. 2019). For the synthesis parameters of the latter $\mathrm{Cu}$ NPs obtained from a scientific sputter deposition (used for comparison issues), see Meischein et al. (Meischein et al. 2019). The sputter rate can change over target usage time. However, this becomes relevant only at the end of the target lifetime when the erosion track becomes deep. The $\mathrm{Cu}$ target was unused before the largescale deposition. The rate was determined directly after the large-scale deposition.
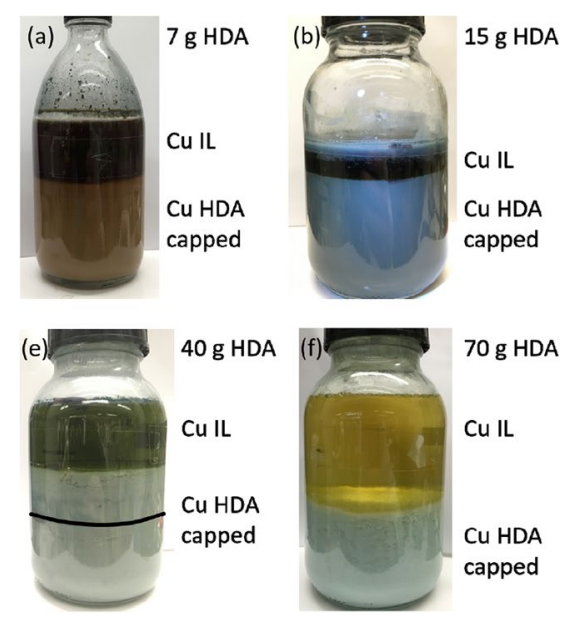

Fig. 1 Different stages of the NP capping process. The amount of HDA-capped NPs increases with the increasing amount of used HDA which is $7 \mathrm{~g}$ in (a), $15 \mathrm{~g}$ in (b), $25 \mathrm{~g}$ in (c), $30 \mathrm{~g}$ in (d), $40 \mathrm{~g}$ in (e), $70 \mathrm{~g}$ in (f), and $80 \mathrm{~g}$ in (g). Between the HDA addition from 7 to $15 \mathrm{~g}$, a bigger bottle had to be used since the volume of capped NPs with the necessary HDA and acetonitrile addition would have exceeded the volume of the smaller bottle. Between images (d) and (e),
Capping of $\mathrm{Cu}$ NPs using HDA as capping agent

Acetonitrile (purity $\geq 99.5 \%$ from Fisher Scientific UK, Loughborough, England) was added to the $\mathrm{Cu} /$ IL suspension until both liquid phases had the same height in the bottle. Then, HDA (purity $>95.0 \%$, from TCI Deutschland GmbH, Eschborn, Germany) was continuously added to the mixture with increasing single portions for slowly starting the NP capping and precipitation process (see Fig. 1). After each addition of capping agent (HDA), the IL acetonitrile mixture was stirred at $1500 \mathrm{rpm}$ for $48 \mathrm{~h}$. With increasing amount of HDA, the strength of the color of the remaining $\mathrm{Cu}$ IL decreases (Fig. 1c). A color change from brown to green of IL and capped NPs occurs due to the reaction of $\mathrm{Cu}$ with oxygen and water since all addition and stirring cycles were performed in air. For extracting all $\mathrm{Cu}$ NPs from the IL and obtaining a transparent IL phase above the NP phase, a total amount of $80 \mathrm{~g}$ HDA had to be used.

\section{Extraction of HDA-capped Cu NPs}

Extraction of the HDA-capped Cu NPs from the IL/ acetonitrile mixture was achieved by centrifugation. The whole amount of IL/acetonitrile mixture was

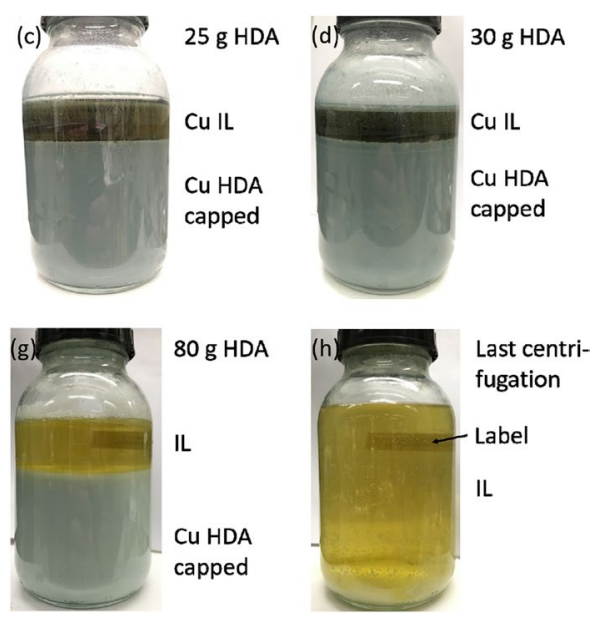

approximately half of the capped Cu NPs was used for the first centrifugation session after reaching $35 \mathrm{~g}$ of HDA in total. The black line in (e) shows the level of the remaining HDA-capped $\mathrm{Cu}$ NPs after the extraction of the NP material. In (h), the remaining liquid after the 6th centrifugation round is shown. The transparency (note the label which is positioned at the back wall of the bottle) indicates that the NPs were extracted from the IL to a very high degree 
filled into centrifuge tubes (total volume $50 \mathrm{~mL}$ ) so that each tube was filled with $35 \mathrm{~mL}$. The rest of the volume was filled with fresh acetonitrile. After that, the tubes were shaken so that both liquid phases were mixed. The centrifugation was conducted using a Sigma 4-16S centrifuge (from Sigma Laborzentrifugen $\mathrm{GmbH}$, Osterode am Harz, Germany) operated at 8000 rotations per minute, corresponding to a relative centrifugal force ( $\mathrm{rcf}$ ) of 10,375 g. After the first centrifugation cycle of $2 \mathrm{~min}$, the mixture of slight yellow/green-colored IL/acetonitrile mixture above the capped $\mathrm{Cu}$ NPs at the bottom of the tubes was removed with a syringe and filled back into the container with the IL/acetonitrile mixture. This was done to ensure that the NP material, which was not capped by the HDA, was not lost during extraction. The tubes were refilled with new acetonitrile and were shaken again as long as the capped NPs were re-dissolved homogeneously in the tube volume. The centrifugation process was conducted again for the second to forth cycle. After a total of 4 cycles with consecutive removal and disposal of the remaining (clear) IL/acetonitrile mixture above the capped NPs, the remaining wet $\mathrm{Cu} \mathrm{NP}$ powder from each centrifugation tube was mixed into acetonitrile and left in an open container overnight so that the acetonitrile could evaporate. The remaining HDA-capped and dried $\mathrm{Cu} \mathrm{NP}$ powder was weighed for determining the extracted NP amount using the measured $\mathrm{Cu}$ metal content evaluated by ICP-MS.

\section{TEM sample preparation}

For the preparation of TEM samples, holey carboncoated Au grids (200 mesh, Plano GmbH, Wetzlar, Germany) were used. For the untreated as-sputtered $\mathrm{Cu}$ IL, $2.5=\mathrm{L}$ suspension was dropped on the carbon-coated grid side and left at this side for adhesion of the NPs for $2.5 \mathrm{~h}$. In order to prevent possible grid contamination originating from interaction of the electron beam with the IL during TEM analysis, the grids were washed dropwise with dried acetonitrile for $1 \mathrm{~h}$ under inert conditions (see supporting information of Meyer et al. (Meyer et al. 2018)) and then stored in Ar atmosphere.

For the TEM investigations of the HDA-capped $\mathrm{Cu}$ NPs, a spatula tip of the centrifuged powder was filled under air in an Eppendorf cap with toluene (purity $\geq$ 99.5\%, from VWR International GmbH, Darmstadt,
Germany) and treated in an ultrasonic bath for $2 \mathrm{~min}$. After the treatment in the ultrasonic bath, the powder was completely dissolved in toluene, resulting in a homogeneous green color of the beforehand colorless liquid, proving the successful re-dispersion of the HDA-capped $\mathrm{Cu}$ NPs into an apolar solvent. A few drops of the obtained mixture were filled into a new Eppendorf cap and were mixed with more toluene. This dilution was necessary to reduce the amount of HDA remaining on the TEM grid. A few drops of the obtained final solution were dropped on the carboncoated side of the TEM grid and left for evaporation of the toluene inside a fume hood. Conventional TEM and high-resolution TEM (HRTEM) studies were performed using a FEI Tecnai F20 S/TEM instrument operated at $200 \mathrm{kV}$. For the analysis of the $\mathrm{Cu}$ NP mean diameter, at least 253 NPs were evaluated manually.

\section{ICP-MS measurements}

For ICP-MS measurements, an amount of two times $50=\mathrm{L}$ of the $\mathrm{Cu} \mathrm{IL}$ and an amount of $13.5 \mathrm{mg}$ of the dried $\mathrm{Cu}$ NP powder obtained from the centrifugations was separated in suitable Teflon containers. To ensure a correct volume of IL separated into the containers, each container with the respective IL inside was additionally weighed. For the $\mathrm{Cu} \mathrm{IL}$, weighs of $54.7 \mathrm{mg}$ in the first loaded and $69.6 \mathrm{mg}$ in the second loaded containers were measured. The differences between first and second loaded containers may be due to the dry pipette tip during the first loading since the viscosity of the IL prevents the complete loading and unloading of the adjusted IL volume in the used Eppendorf pipette tip. The separated samples were diluted each with $4 \mathrm{~mL}$ of $69 \%$ concentrated phosphoric acid of the "ROTIPURAN® Supra" line. These mixtures were chemically digested in a Multiwave Pro microwave digestion device with 8-slot container holder 8NXF100 (Anton Paar GmbH, Graz, Austria). The digestion occurred at maximum temperature $240^{\circ} \mathrm{C}$ and maximum pressure 60 bar to ensure a complete transfer of the investigated material into solvable nitrides which could be measured in the ICP-MS. The resulting solutions were further diluted with ultrapure water (conductivity $0.055 \rightleftharpoons \mathrm{S}$ $/ \mathrm{cm}$ ) to a total volume of $10 \mathrm{~mL}$ per sample. From this stem-solutions, 1:100- and 1:1000-dissolutions were produced and experienced an acidification with 
$2 \%$ phosphoric acid prior to the measurements in an iCAP RQ ICP-MS device (from Thermo Fisher Scientific Inc., Waltham, MA, USA). The ICP-MS measurements were conducted in the KED-mode to decrease disturbances from molecule ions.

\section{Results}

The results are presented in a TEM part, confirming the existence of NPs in the sputtered ILs as well as the extracted powder and a general analysis part of the $\mathrm{Cu}$ NP extraction in terms of necessary capping agent, obtained extracted material, and its metal content determined by ICP-MS.

TEM results of the NPs obtained from the ILs and the extracted powder

As-prepared and HDA-treated ILs were analyzed by TEM to confirm the existence of Cu NPs in the IL and the HDA/IL/acetonitrile mixture. The TEM data of the $\mathrm{Cu}$ NPs obtained from the upscaled sputtering in IL were compared with TEM data of Cu NPs synthesized with our standard procedure (Meischein et al. 2019) for scientific investigations. Figure 2 shows TEM images of $\mathrm{Cu}$ NPs obtained from the upscaled synthesis $\mathrm{Cu} / \mathrm{IL}$ directly after the deposition. The main amount of the NPs is organized in group structures where the individual NPs are close together. Crystallinity of the NPs was confirmed by the FFT data of the NPs depicted in the HRTEM image insets.

$\mathrm{Cu}$ NPs synthesized with our standard protocol for scientific purposes are shown in Fig. 3, again directly after sputter deposition in IL. These NPs show a very uniform distribution on the TEM grid and bigger NPs with respect to the upscaled-synthesized NPs shown in Fig. 2. For both deposition types, the NPs show the fcc crystal structure as analyzed by the respective FFT data.

Furthermore, the size distributions of the NPs are comparable, as shown in Fig. 4. In both NP systems, the highest number of NPs has a diameter of $2 \mathrm{~nm}$, followed by $3 \mathrm{~nm}$ diameter. Additionally, the mean diameters are comparable. For the upscaled deposition, a NP mean diameter of $(2.6 \pm 1.1) \mathrm{nm}$ was evaluated which is in good agreement with the mean diameter of $(2.8 \pm 1.1) \mathrm{nm}$ for the "scientific deposition."

The comparison between the HDA-capped $\mathrm{Cu}$ NPs in Fig. 5 and the as-prepared $\mathrm{Cu}$ NPs from the upscaled deposition in Fig. 2 shows that the HDA capping separated the NPs from each other and prohibited the formation of the agglomeration of $\mathrm{Cu}$ NPs. However, for both sample conditions, single NPs have been found in TEM investigations, with sizes around $2 \mathrm{~nm}$.

General analysis of the extracted $\mathrm{Cu}$ NP powder

For the final state of the extraction procedure depicted in Fig. 1g, a total amount of $80 \mathrm{~g}$ HDA had to be used for extracting all of the NP material and leaving the IL nearly transparent. The centrifugation resulted in a total amount of capped NPs of approximately $139.32 \mathrm{~g}$. During the precipitation procedure, no agglomeration and precipitation of the NPs besides when capping with HDA were observed, as depicted in the image series of Fig. 1. Only the brown/green HDA-capped $\mathrm{Cu}$ NPs segregate out of the liquid IL, but no other segregates have been discovered. The liquid phase above the capped NPs becomes more and more transparent with increased HDA addition, but the homogeneous color indicates that the NPs stay stabilized in the IL matrix without own agglomeration.

The ICP-MS results revealed the actual content of the sputtered $\mathrm{Cu}$ material in the respective stabilizing medium (IL and HDA capping) since this technique is capable of identifying even traces of elements ( $\mathrm{pg} / \mathrm{mL}$ to high $=\mathrm{g} / \mathrm{mL}$ scales) in large sample volumes (Nelms 2005). For the investigation of the IL, $50=\mathrm{L}$ of the large-scale $\mathrm{Cu}$ IL was investigated. The measured $\mathrm{Cu}$ weight proportion was $0.922 \%$, which means the total IL volume of $50 \mathrm{~mL}$ would contain $0.66 \mathrm{~g}$ of sputtered $\mathrm{Cu}$ (using the assumption of $1.44 \mathrm{~g} / \mathrm{cm}^{3}$ for the IL density as outlined by the IL delivering company) when projecting the ICP-MS result on the whole sputtering process. For the investigation of the HDA/Cu powder, $13.5 \mathrm{mg}$ of the centrifuged and dried powder were analyzed, resulting in a $\mathrm{Cu}$ weight proportion of $0.818 \%$. The projection on the whole amount of the powder means that it contains $1.14 \mathrm{~g} \mathrm{Cu}$ originating from the sputtering process. 
Fig. 2 Overview of NPs from the as-prepared upscaled-synthesized $\mathrm{Cu}$ IL directly after deposition at different magnifications $((\mathbf{a}, \mathbf{b})$ at $97000 \mathrm{x},(\mathbf{c}, \mathbf{d})$ at 195000x, (e) at 450000x and (f) $790000 x$ ). The $\mathrm{Cu}$ NPs are organized mainly in loosely bounded agglomeration structures with individual NPs. Crystallinity was detected as depicted in the close-up NP image in (f) and the corresponding FFT graph in the inset
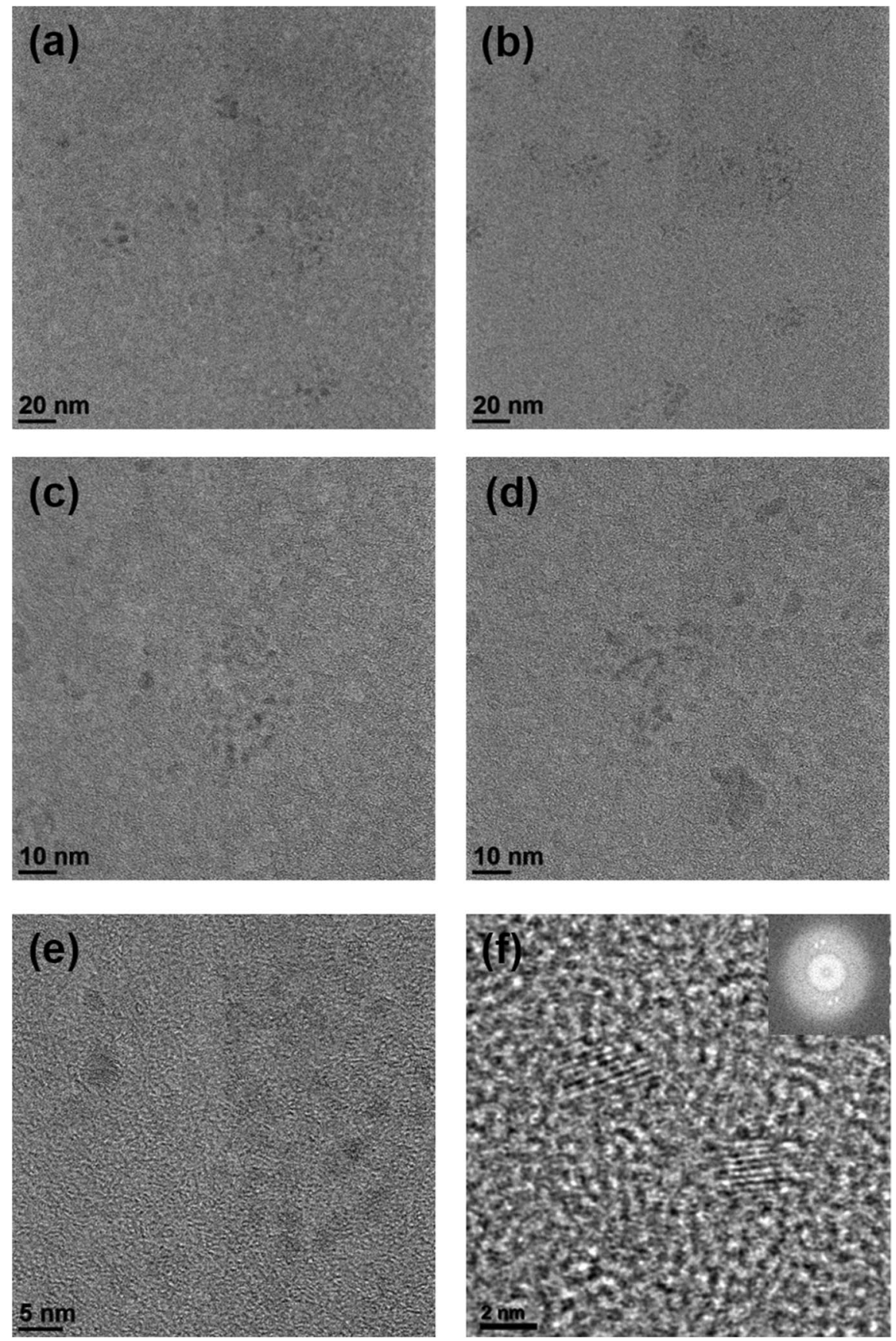

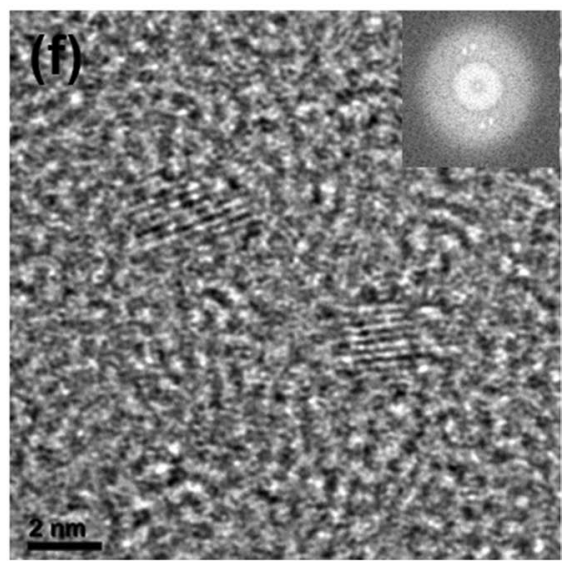

\section{Discussion}

The aim of this study was to clarify the question, if an upscaled synthesis of NPs in IL could be achieved by sputter deposition. According to literature, a NP weight in the double-digit gram regime can be regarded as large-scale NP synthesis (Lam et al. 2000; Cui et al. 2009; Song and Chen 2003). As reported in literature, weights of the NP materials obtained from different large-scale synthesis methods range from $2 \mathrm{~g}$ (ball milling) (Lam et al. 2000) to $40 \mathrm{~g}$ (chemical approach) (Park et al. 2004) with synthesis times 
Fig. 3 Overview of NPs from the as-prepared $\mathrm{Cu}$ IL synthesized for scientific purposes directly after deposition at different magnifications $((\mathbf{a}, \mathbf{b})$ at $97000 x,(\mathbf{c}, \mathbf{d})$ at $145000 \mathrm{x}$, (e) at 450000x and (f) at 790000x). Crystallinity was detected as depicted in the close-up NP image in (f) and the corresponding FFT graph in the inset
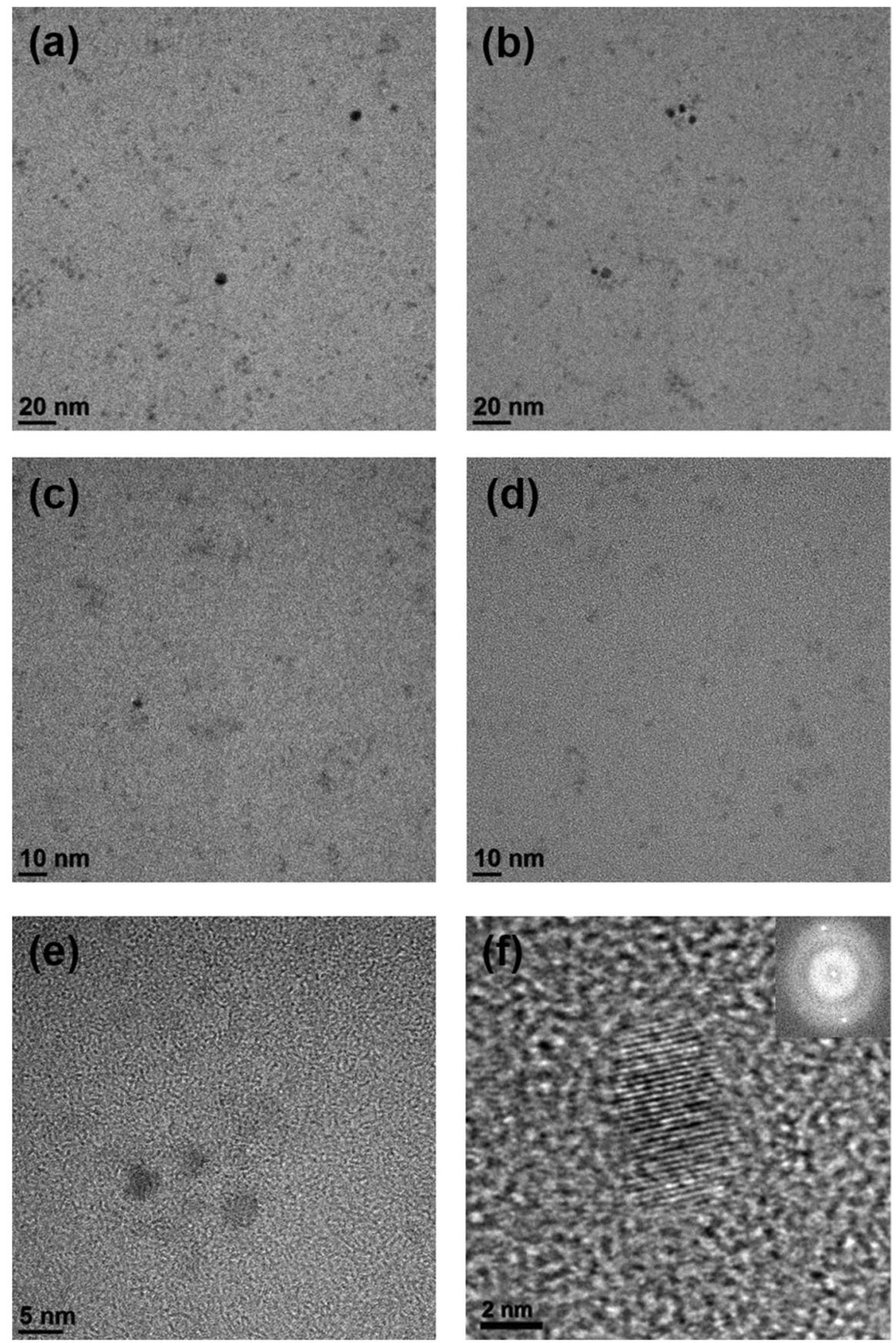

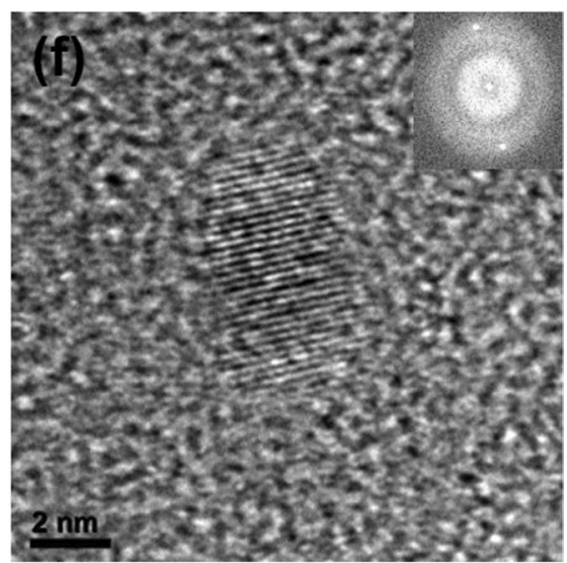

ranging from $4 \mathrm{~h}$ and $30 \mathrm{~min}$ (chemical approach) to 7-10 days with additional $50 \mathrm{~h}$ of further preparation (ball milling). However, the chemical and the ball milling processes require several preparation steps and in the case of the chemical approach different chemicals whereas for sputtering in IL, only the target and the sputter system with the process gas are necessary. From the deposition rate and time one can calculate the nominal film thickness of a $\mathrm{Cu}$ film deposited on the IL surface. The comparison of our upscaled $\mathrm{Cu}$ deposition with the $\mathrm{Cu}$ deposition for scientific purposes shows some interesting differences 
(a)

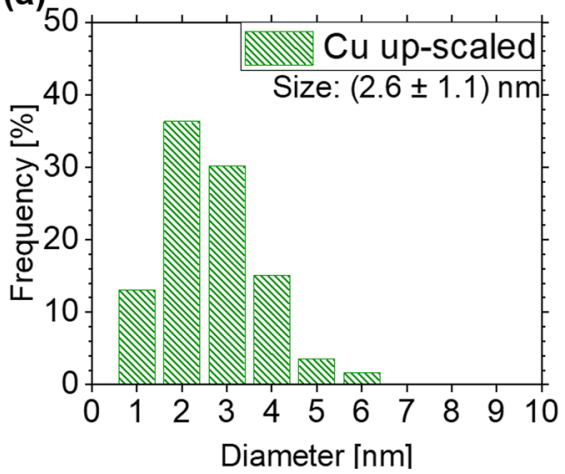

Fig. 4 NP size distributions for both types of $\mathrm{Cu}$ NP in IL depositions. The NP size distribution of the upscaled $\mathrm{Cu}$ deposition is shown in (a) and the size distribution of the NPs obtained from the scientific deposition is depicted in (b). Both

(see Table 1) in terms of the obtained sputtered film and the IL loaded with NPs.

The sample holders for both deposition types are designed so that the IL volume is cylindrical with circular IL surfaces. For comparison issues, only one cavity of the cavity array plate used as sample holder for the scientific depositions has been considered. This limit was chosen since the concentration of the sputtered material (considered crucial parameter for (i) demonstrating the difference in terms of sputtered material stabilized in the ILs and (ii) for estimating if all of the sputtered material can be stabilized in the IL) will not change if more than one cavity is used.

The calculated film thickness with the information of the IL surface diameter results in a calculated upper weight limit of $\mathrm{Cu}$ sputtered into the IL. In the case of the upscaled synthesis, a $\mathrm{Cu}$ amount of approximately $1 \mathrm{~g}$ is deposited on and into the IL. Since there was no agglomeration of the $\mathrm{Cu}$ material in the IL volume, the amount of $1 \mathrm{~g} \mathrm{Cu}$ is assumed to be stabilized as well-distributed NPs in the IL (see Fig. 2). Additionally, the size distributions of the upscaled and the scientific depositions show a good agreement concerning mean diameter and general NP sizes, also indicating no relevant quality difference of the upscaled and scientifically synthesized ILs.

The ICP-MS results support this assumption. For the upscaled $\mathrm{Cu}$ deposition, a $\mathrm{Cu}$ amount of $0.66 \mathrm{~g}$ in the IL and of $1.14 \mathrm{~g}$ in the extracted powder was extrapolated. The discrepancies of the respective metal amounts in the measured ILs from the (b)

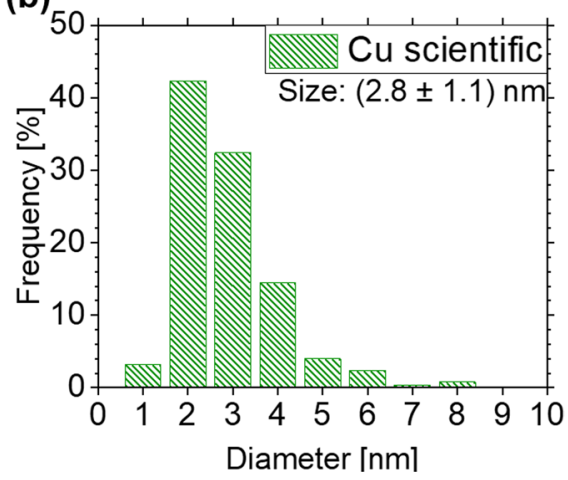

size distributions show a comparable spread of the NP diameters with the NPs from the scientific deposition being slightly bigger and a maximum NP size exceeding the maximum NP size obtained from the upscaled $\mathrm{Cu}$ deposition

calculated values may be explained by the pipetting and weighing of the ILs, since always a small amount of IL stays in the pipette tip and since the high viscosity of the IL complicates the incorporation of exact 50 $\rightleftharpoons$ L from the container into the pipette and from there into the Teflon container for the chemical digestion. The $\mathrm{Cu}$ amount extrapolated from ICP-MS results for the centrifuged $\mathrm{HDA} / \mathrm{Cu}$ powder is in agreement with the calculated $\mathrm{Cu}$ amount deposited into the IL and lies within a tolerance of $10 \%$ error. Comparing now the results from Table 1 with the amounts of NP material and the necessary synthesis times for chemical (up to $40 \mathrm{~g}$ in $4.5 \mathrm{~h}$ ) and mechanical ( $2 \mathrm{~g}$ in 7-12 days) approaches, the large-scale NP synthesis with our approach lies in between mechanical and chemical ways in terms of amount per time unit, but shows the advantages of no necessary additional chemicals or synthesis procedures besides the actual sputtering process.

Although the $\mathrm{Cu}$ metal concentration in the upscaled-synthesized IL is five times higher than the $\mathrm{Cu}$ metal concentration in the scientific synthesis IL, well-dispersed and non-agglomerated NPs are obtained in both cases. For the upscaled synthesis, the concentration of sputtered material in the ILs is now a parameter to estimate if the NPs stay well-dispersed in the stabilizing IL matrix without agglomerations. Most publications report on NPs in ILs synthesized with methods other than sputtering with concentrations in the range of our scientific IL (Richter et al. 2011; Salas et al. 2011; Zhang et al. 2012). Also, 
Fig. 5 Overview of the HDA-capped $\mathrm{Cu}$ NPs dissolved in toluene prior to the TEM grid preparation at different magnifications $((\mathbf{a}, \mathbf{b})$ at $97000 \mathrm{x},(\mathbf{c}, \mathbf{d})$ at $195000 x,(e)$ at $450000 x$ and (f) at $690000 x)$. Compared to the as-prepared $\mathrm{Cu} \mathrm{NPs}$, the HDA-capped NPs are not organized in agglomerated structures but are well-distributed
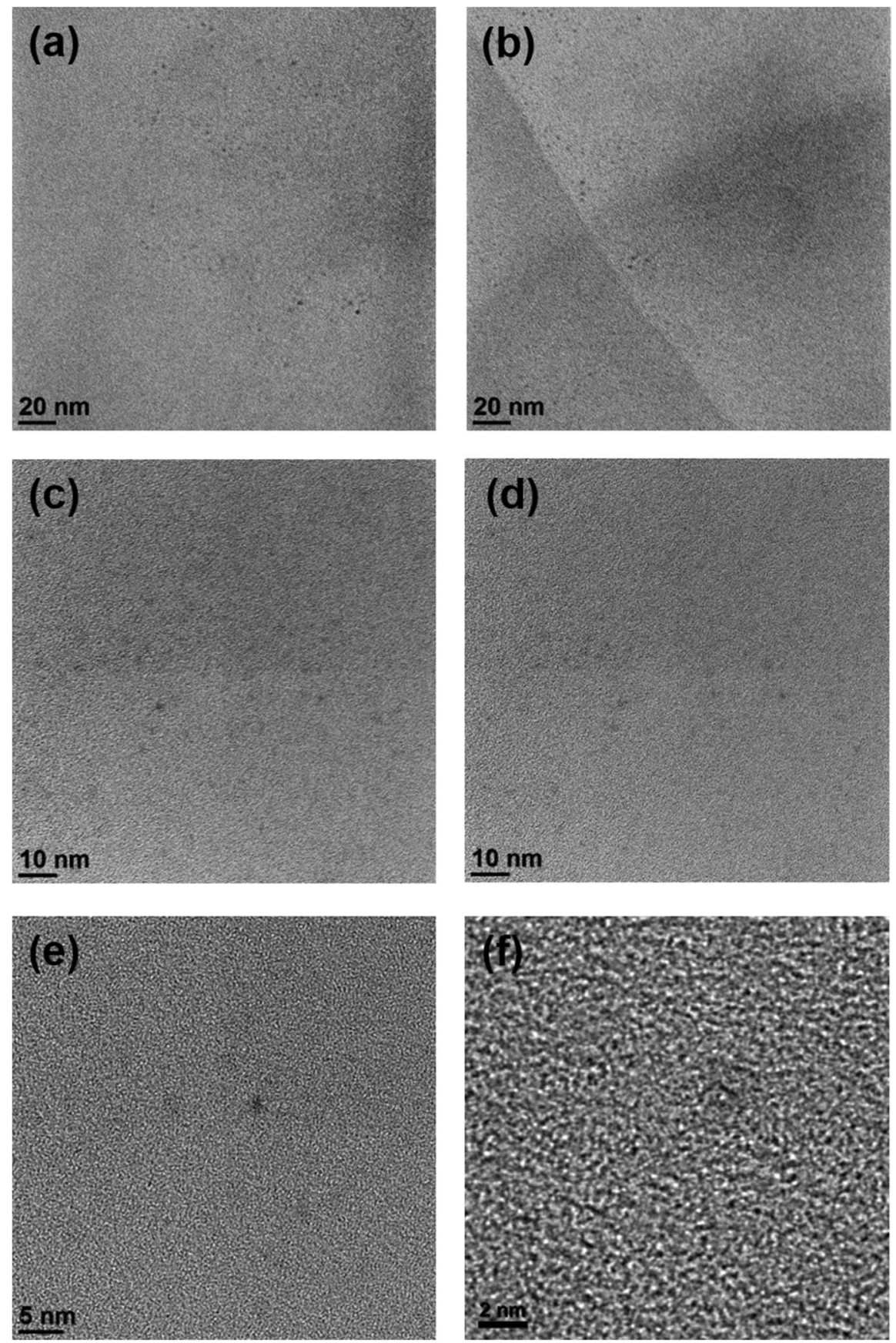

Table 1 Comparison of the deposition parameters and sample holder characteristics of the upscaled and the scientific Cu deposition in IL

\begin{tabular}{llllllll}
\hline Deposition & $\begin{array}{l}\text { Deposition } \\
\text { rate }(\mathrm{nm} / \mathrm{s})\end{array}$ & $\begin{array}{l}\text { Deposition } \\
\text { time }(\mathrm{h})\end{array}$ & $\begin{array}{l}\text { Film thick- } \\
\text { ness }(\mu \mathrm{m})\end{array}$ & $\begin{array}{l}\text { Diameter IL } \\
\text { surface }(\mathrm{mm})\end{array}$ & $\begin{array}{l}\text { Calculated film } \\
\text { weight }(\mathrm{g})\end{array}$ & $\begin{array}{l}\text { IL volume }(\mathrm{mL}) \\
\begin{array}{l}\text { Cu concen- } \\
\text { tration in IL } \\
(\mathrm{mol} / \mathrm{L})\end{array}\end{array}$ \\
\hline Upscaled & 0.143 & 24 & 12.364 & 110 & 1.05 & 50 & 0.3313 \\
"Scientific" & 0.080 & 2 & 0.576 & 6 & 0.000146 & 0.035 & 0.0656 \\
\hline
\end{tabular}


concentrations in the range of our upscaled IL (Qadir et al. 2019, 2018) and a concentration higher than in our upscaled IL was reported (with a small additive of IL-like ions originating from the IL synthesis process, see supporting information of Dash and Scott) (Dash and Scott 2009). Those results show that NP concentrations even higher than our obtained concentration are possible without uncontrolled or unwanted NP agglomeration. The absence of the formation of a $\mathrm{Cu}$ film on the IL surface and the missing of unwanted $\mathrm{Cu}$ agglomerations within the uninfluenced $\mathrm{IL}$ after the depositions reveals that the stabilization ability of the IL was not exceeded by our upscaled synthesis. Therefore, the amount of stabilized NPs in IL can be enlarged easily, since we sputtered with a relatively small cathode for a limited time into a limited volume of IL. Until now, reports in the literature comprising sputtering onto ILs for the large-scale synthesis of NPs are missing. Thus, we want to underline the novelty of our approach to demonstrate the capability of a successful large-scale NP synthesis by sputtering in IL without unwanted NP agglomeration and good NP quality if the usual sputter process is modified.

For example, sputtering with more than one cathode would increase the sputtering rate by the number of the used cathodes and thus lead to more NP material due to a nominally thicker "virtual" film. Sputtering from bigger cathodes would also increase the deposition rate since (i) the target area exposed to the sputtering Ar gas is bigger and (ii) the applied sputtering power can be increased for bigger targets since the maximum power before damaging the target increases with the target area and volume. Finally, if the used IL volume is increased, also more NPs can be stabilized. Due to the current available technical equipment, it would be possible to design sputtering systems with a consecutive IL supply from ambient pressure to required sputter pressures in the sputtering area. A possible schematic design of an upscaled product unit is shown in Fig. 6: an IL assembly line where the IL is (purified and) loaded on the line in Ar atmosphere, pumped down over several consecutive pumping stations to the sputter pressure and is released after sputtering again to an Ar (or any other suitable inert) atmosphere at ambient pressure for storing and packing the IL product.

The quality of the obtained NPs shows no relevant differences concerning shape and distribution with respect to NPs synthesized for scientific purposes. The upscaled-synthesized NPs exist in the as-sputtered and the HDA-capped state as individual, nonagglomerated particles, as proven by the TEM investigations. However, in the HDA-capped state, the NPs have a smoother and more regular distribution as in the as-prepared state. This distribution is attributed to the HDA capping agent, which is extracting the capped NPs out of the IL. The comparable long apolar alkyl chain of the HDA prevents physical contact of the NPs and contributes to a comparable uniform distribution over the TEM grid, since the chains at one capped NP also serve as distance keepers to the surrounding capped NPs. (a)

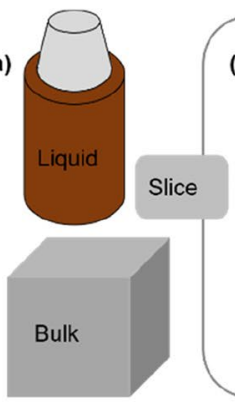

Fig. 6 Schematic illustration of a possible upscaling production line for NP/IL. IL can be delivered in liquid or bulk state in (a) and introduced into the loading unit (b) with inert atmosphere. The IL can be purified within the loading unit, e.g., by annealing at mild temperatures below $100^{\circ} \mathrm{C}$ within slight vacuum and then loaded onto the assembly line. The assembly line is pumped at different pumping stages in (c) until the sput- ter pressure is reached. The sputter unit (d) is directly attached to the pumping unit. The sputtered IL is transported back to atmospheric pressure via the venting unit (e) using inert atmosphere. The vented assembly line ends in the packing unit (f) also among inert atmosphere where the NP/IL suspension is packed or transported to its further applications 
By extracting the $\mathrm{Cu}$ NPs from the IL with the HDA capping agent, more applications become possible, e.g., if ILs were not an appropriate solvent for the desired process catalyzed by the NPs. The capped NPs can be easily dissolved back into apolar solvents as shown by the successful solvation into toluene, increasing the pool of suitable solvents for the NP colloids.

Since either NPs in IL can be used for their desired applications within the stabilizing IL or the NPs could be extracted by chemical routes, the obtained upscaled-synthesized NP amount can be used for a various number of applications now with industrial scales.

\section{Conclusion}

Sputtering was applied for an upscaled synthesis of $\mathrm{Cu}$ NPs in IL. The comparison of $\mathrm{Cu}$ NPs obtained from the upscaled synthesis route with $\mathrm{Cu}$ NPs obtained from scientific preparation protocols shows only subtle differences although the material concentration in the upscaled IL is five times higher than in the scientific IL. The extraction of the Cu NPs obtained from the upscaled synthesis by addition of HDA as capping agent was performed for showing an alternative way of NP stabilization as well as demonstrating that those NPs can be used in different solvents than in ILs. The result of about $1 \mathrm{~g}$ of stabilized $\mathrm{Cu}$ NPs from sputtering in IL with special parameters but in a comparable small IL volume using a small cathode with low sputtering power shows the suitability of sputter deposition into ILs for large-scale NP synthesis when using scaled synthesis machines and parameters. In comparison with other approaches for an upscaled NP synthesis, sputtering onto ILs lies in between mechanical and chemical techniques in terms of produced amount per time. However, the sputter synthesis comprises only the actual sputter process to obtain the stabilized NPs without the necessity of additional chemicals or synthesis processes in contrast to other methods. Thus, for example, simply increasing the deposition rate onto a larger IL volume would increase the obtained nanomaterial amount by the factor of the increased rate, underlining the feasibility of sputtering conducted for a large-scale NP synthesis.
Acknowledgements We want to thank Florian Lourens for assistance in literature review concerning nanoparticle upscaling. Additionally, we want to thank the Center of Electrochemical Sciences (CES) at the Ruhr University Bochum for (i) granting access to their centrifuge and Dr. Patrick Wilde for his introduction into the centrifuge operation and (ii) for conducting the ICP-MS measurements operated by Martin Trautmann. CES: Chair for Analytical Chemistry, Center for Electrochemical Sciences, Faculty of Chemistry and Biochemistry, Ruhr University Bochum, Universitätsstr. 150, D-44780 Bochum, Germany.

Author contribution Michael Meischein conducted the sample synthesis, the sample processing, the TEM sample analysis, the NP size determination, all experiments except those mentioned in the acknowledgements and wrote the manuscript. Alfred Ludwig provided funding, scientific supervision and discussion, validation, and manuscript review and editing. All authors have read and agreed to the published version of the manuscript.

Funding Open Access funding enabled and organized by Projekt DEAL. This work was funded by the BMBF project NEMEZU and the German Science Foundation (DFG) via the project LU1175/23-1.

Data availability Not applicable.

Code availability Not applicable.

\section{Declarations}

Ethics approval Not applicable.

Consent to participate Not applicable.

\section{Consent for publication Not applicable.}

Conflict of interest The authors declare no competing interests.

Open Access This article is licensed under a Creative Commons Attribution 4.0 International License, which permits use, sharing, adaptation, distribution and reproduction in any medium or format, as long as you give appropriate credit to the original author(s) and the source, provide a link to the Creative Commons licence, and indicate if changes were made. The images or other third party material in this article are included in the article's Creative Commons licence, unless indicated otherwise in a credit line to the material. If material is not included in the article's Creative Commons licence and your intended use is not permitted by statutory regulation or exceeds the permitted use, you will need to obtain permission directly from the copyright holder. To view a copy of this licence, visit http://creativecommons.org/licenses/by/4.0/. 


\section{References}

Athanassiou EK, Grass RN, Stark WJ (2006) Large-scale production of carbon-coated copper nanoparticles for sensor applications. Nanotechnology 17:1668-1673. https://doi. org/10.1088/0957-4484/17/6/022

Astruc D (2008) Nanoparticles and catalysis. Wiley-VCH, Weinheim

Al-Madanat O, AlSalka Y, Ramadan W, Bahnemann DW (2021) TiO2 photocatalysis for the transformation of aromatic water pollutants into fuels. Catalysts 11:317-361. https://doi.org/10.3390/catal11030317

Baraton M-I (ed) (2003) Synthesis, functionalization and surface treatment of nanoparticles. American Scientific Publ, Stevenson Ranch, Calif

Bracey CL, Ellis PR, Hutchings GJ (2009) Application of copper-gold alloys in catalysis: current status and future perspectives. Chem Soc Rev 38:2231-2243. https://doi.org/ 10.1039/b817729p

Breisch M, Grasmik V, Loza K, Pappert K, Rostek A, Ziegler N, Ludwig A, Heggen M, Epple M, Tiller JC et al (2019) Bimetallic silver-platinum nanoparticles with combined osteo-promotive and antimicrobial activity. Nanotechnology 30:305101. https://doi.org/10.1088/1361-6528/ab172 $\mathrm{b}$

Cui H, Feng Y, Ren W, Zeng T, Lv H, Pan Y (2009) Strategies of large scale synthesis of monodisperse nanoparticles. Recent Pat Nanotechnol 3:32-41. https://doi.org/10.2174/ 187221009787003302

Conde J, Doria G, Baptista P (2012) Noble metal nanoparticles applications in cancer. Journal of Drug Delivery 2012:751075. https://doi.org/10.1155/2012/751075

Chen L, Hu H, Chen Y, Gao J, Li G (2021) Plasmonic Cu 2-X $\mathrm{S}$ nanoparticles: a brief introduction of optical properties and applications. Mater Adv 2:907-926. https://doi.org/ 10.1039/D0MA00837K

Datta MK, Pabi SK, Murty BS (2000) Phase fields of nickel silicides obtained by mechanical alloying in the nanocrystalline state. J Appl Phys 87:8393-8400. https://doi.org/ $10.1063 / 1.373553$

Dash, P.; Scott, R. W. J. (2009) 1-Methylimidazole stabilization of gold nanoparticles in imidazolium ionic liquids. Chemical communications (Cambridge, England): 812-814. https://doi.org/10.1039/B816446K

Dupont J, Scholten JD (2010) On the structural and surface properties of transition-metal nanoparticles in ionic liquids. Chem Soc Rev 39:1780-1804. https://doi.org/10. 1039/b822551f

Garzón-Manjón, A.; Solano, E.; La Mata, M. de; Guzmán, R.; Arbiol, J.; Puig, T.; Obradors, X.; Yáñez, R.; Ricart, S.; Ros, J. (2015) Induced shape controllability by tailored precursor design in thermal and microwave-assisted synthesis of Fe3O4 nanoparticles. J Nanopart Res 17. https:// doi.org/10.1007/s11051-015-3070-x

Garzón-Manjón A, Aranda-Ramos A, Melara-Benítez B, Bensarghin I, Ros J, Ricart S, Nogués C (2018a) Simple synthesis of biocompatible stable $\mathrm{CeO} 2$ nanoparticles as antioxidant agents. Bioconjug Chem 29:2325-2331. https:// doi.org/10.1021/acs.bioconjchem. 8 b00300
Garzón-Manjón A, Meyer H, Grochla D, Löffler T, Schuhmann W, Ludwig A, Scheu C (2018b) Controlling the amorphous and crystalline state of multinary alloy nanoparticles in an ionic liquid. Nanomaterials (basel, Switzerland) 8(11):903. https://doi.org/10.3390/nano8110903

Ghotekar S, Pansambal S, Bilal M, Pingale SS, Oza R (2021) Environmentally friendly synthesis of $\mathrm{Cr} 2 \mathrm{O} 3$ nanoparticles: characterization, applications and future perspective-a review. Case Studies in Chemical and Environmental Engineering 3:100089. https://doi.org/10. 1016/j.cscee.2021.100089

Holbrey JD, Seddon KR (1999) Ionic liquids. Clean Technol Environ Policy 1:223-236. https://doi.org/10.1007/s1009 80050036

Hatakeyama Y, Okamoto M, Torimoto T, Kuwabata S, Nishikawa K (2009) Small-angle X-ray scattering study of $\mathrm{Au}$ nanoparticles dispersed in the ionic liquids 1-alkyl3-methylimidazolium Tetrafluoroborate. J Phys Chem C 113:3917-3922. https://doi.org/10.1021/jp807046u

Hirano M, Enokida K, Okazaki K-I, Kuwabata S, Yoshida H, Torimoto T (2013) Composition-dependent electrocatalytic activity of AuPd alloy nanoparticles prepared via simultaneous sputter deposition into an ionic liquid. Phys Chem Chem Phys 15:7286-7294. https://doi.org/ $10.1039 / \mathrm{c} 3 \mathrm{cp} 50816 \mathrm{a}$

Itoh H, Naka K, Chujo Y (2004) Synthesis of gold nanoparticles modified with ionic liquid based on the imidazolium cation. J Am Chem Soc 126:3026-3027. https:// doi.org/10.1021/ja039895g

Javed B, Ikram M, Farooq F, Sultana T, Mashwani Z-U-R, Raja NI (2021) Biogenesis of silver nanoparticles to treat cancer, diabetes, and microbial infections: a mechanistic overview. Appl Microbiol Biotechnol 105:22612275. https://doi.org/10.1007/s00253-021-11171-8

Karmakar S, Kumar S, Rinaldi R, Maruccio G (2011) Nanoelectronics and spintronics with nanoparticles. J Phys Conf Ser 292:12002. https://doi.org/10.1088/17426596/292/1/012002

König D, Richter K, Siegel A, Mudring A-V, Ludwig A (2014) High-throughput fabrication of $\mathrm{Au}-\mathrm{Cu}$ nanoparticle libraries by combinatorial sputtering in ionic liquids. Adv Funct Mater 24:2049-2056. https://doi.org/ 10.1002/adfm. 201303140

Kuwabata, S.; Torimoto, T.; Imanishi, A.; Tsu, T. (2014) Use of ionic liquid under vacuum conditions. In: Kadokawa, J.-i. (ed) Ionic liquids-new aspects for the future; Croatia, Japan; pp 597-615

Kim D-Y, Suk Sung J, Kim M, Ghodake G (2015) Rapid production of silver nanoparticles at large-scale using gallic acid and their antibacterial assessment. Mater Lett 155:62-64. https://doi.org/10.1016/j.matlet.2015.04.138

Kharisov BI, Dias HR, Kharissova OV (2019) Mini-review: ferrite nanoparticles in the catalysis. Arab J Chem 12:12341246. https://doi.org/10.1016/j.arabjc.2014.10.049

Khurana K, Jaggi N (2021) Localized surface plasmonic properties of $\mathrm{Au}$ and $\mathrm{Ag}$ nanoparticles for sensors: a review. Plasmonics. https://doi.org/10.1007/ s11468-021-01381-1

Lam C, Zhang YF, Tang YH, Lee CS, Bello I, Lee ST (2000) Large-scale synthesis of ultrafine Si nanoparticles by ball 
milling. J Cryst Growth 220:466-470. https://doi.org/10. 1016/S0022-0248(00)00882-4

Lee Y, Lee J, Bae CJ, Park J-G, Noh H-J, Park J-H, Hyeon T (2005) Large-scale synthesis of uniform and crystalline magnetite nanoparticles using reverse micelles as nanoreactors under reflux conditions. Adv Funct Mater 15:503509. https://doi.org/10.1002/adfm.200400187

Lee C-C, Chen D-H (2006) Large-scale synthesis of Ni-Ag core-shell nanoparticles with magnetic, optical and antioxidation properties. Nanotechnology 17:3094-3099. https://doi.org/10.1088/0957-4484/17/13/002

Lee Y, Choi J-R, Lee KJ, Stott NE, Kim D (2008) Large-scale synthesis of copper nanoparticles by chemically controlled reduction for applications of inkjet-printed electronics. Nanotechnology 19:415604. https://doi.org/10. 1088/0957-4484/19/41/415604

Li J, Zhang JZ (2009) Optical properties and applications of hybrid semiconductor nanomaterials. Coord Chem Rev 253:3015-3041. https://doi.org/10.1016/J.CCR.2009.07. 017

Lu J, Yang J-X, Wang J, Lim A, Wang S, Loh KP (2009) Onepot synthesis of fluorescent carbon nanoribbons, nanoparticles, and graphene by the exfoliation of graphite in ionic liquids. ACS Nano 3:2367-2375. https://doi.org/10.1021/ nn900546b

Luska KL, Migowski P, Leitner W (2015) Ionic liquid-stabilized nanoparticles as catalysts for the conversion of biomass. Green Chem 17:3195-3206. https://doi.org/10. 1039/C5GC00231A

Löffler T, Meyer H, Savan A, Wilde P, GarzónManjón A, Chen Y-T, Ventosa E, Scheu C, Ludwig A, Schuhmann W (2018) Discovery of a multinary noble metal-free oxygen reduction catalyst. Adv Energy Mater 8:1802269. https:// doi.org/10.1002/aenm.201802269

Liu Y, Xu X, Liu K, Liu H (2020) Synthesis of Cu 2 ZnSnSe 4 thin-film solar cells from nanoparticles by a non-vacuum mechanical ball milling and rapid thermal processing. Micro \& Nano Letters 15:887-891. https://doi.org/10. 1049/mnl.2019.0788

Migowski P, Dupont J (2007) Catalytic applications of metal nanoparticles in imidazolium ionic liquids. Chemistry (weinheim an Der Bergstrasse, Germany) 13:32-39. https://doi.org/10.1002/chem.200601438

Meshesha, B. T.; Barrabés, N.; Medina, F.; Sueiras, J. (2009) Polyol mediated synthesis \& characterization of $\mathrm{Cu}$ nanoparticles: effect of 1-hexadecylamine as stabilizing agent. In: Proceedings of the 1st WSEAS international conference on Nanotechnology; pp 87-91

Meyer H, Meischein M, Ludwig A (2018) Rapid assessment of sputtered nanoparticle ionic liquid combinations. ACS Comb Sci 20:243-250. https://doi.org/10.1021/acsco mbsci.8b00017

Meischein M, Garzón-Manjón A, Frohn T, Meyer H, Salomon S, Scheu C, Ludwig A (2019) Combinatorial synthesis of binary nanoparticles in ionic liquids by cosputtering and mixing of elemental nanoparticles. ACS Comb Sci 21:743-752. https://doi.org/10.1021/acscombsci.9b00140

Meischein M, Fork M, Ludwig A (2020) On the effects of diluted and mixed ionic liquids as liquid substrates for the sputter synthesis of nanoparticles. Nanomaterials (basel,
Switzerland) 10(3):525. https://doi.org/10.3390/nano1 0030525

Nelms SM (2005) Inductively coupled plasma mass spectrometry handbook. Blackwell Publishing Ltd, Oxford, UK

Nguyen MT, Yonezawa T (2018) Sputtering onto a liquid: interesting physical preparation method for multi-metallic nanoparticles. Sci Technol Adv Mater 19:883-898. https://doi.org/10.1080/14686996.2018.1542926

Okazoe S, Kusada K, Wu D, Yamamoto T, Toriyama T, Matsumura S, Kawaguchi S, Kubota Y, Kitagawa H (2020) Synthesis of Mo and Ru solid-solution alloy NPs and their hydrogen evolution reaction activity. Chem Commun (camb) 56:14475-14478. https://doi.org/10.1039/d0cc0 $5958 \mathrm{~g}$

Park J, An K, Hwang Y, Park J-G, Noh H-J, Kim J-Y, Park J-H, Hwang N-M, Hyeon T (2004) Ultra-large-scale syntheses of monodisperse nanocrystals. Nat Mater 3:891-895. https://doi.org/10.1038/nmat1251

Plechkova NV, Seddon KR (2008) Applications of ionic liquids in the chemical industry. Chem Soc Rev 37:123-150. https://doi.org/10.1039/b006677j

Qadir MI, Weilhard A, Fernandes JA, Pedro I, de; Vieira, B. J. C., Waerenborgh, J. C., Dupont, J. (2018) Selective carbon dioxide hydrogenation driven by ferromagnetic RuFe nanoparticles in ionic liquids. ACS Catal 8:1621-1627. https://doi.org/10.1021/acscatal.7b03804

Qadir MI, Bernardi F, Scholten JD, Baptista DL, Dupont J (2019) Synergistic CO2 hydrogenation over bimetallic Ru/ Ni nanoparticles in ionic liquids. Appl Catal B 252:10-17. https://doi.org/10.1016/j.apcatb.2019.04.005

Raut D, Wankhede K, Vaidya V, Bhilare S, Darwatkar N, Deorukhkar A, Trivedi G, Salunkhe M (2009) Copper nanoparticles in ionic liquids: recyclable and efficient catalytic system for 1,3-dipolar cycloaddition reaction. Catal Commun 10:1240-1243. https://doi.org/10.1016/j.catcom. 2009.01.027

Richter K, Birkner A, Mudring A-V (2010) Stabilizer-free metal nanoparticles and metal-metal oxide nanocomposites with long-term stability prepared by physical vapor deposition into ionic liquids. Angew Chem Int Ed 49:2431-2435. https://doi.org/10.1002/anie.200901562

Richter K, Birkner A, Mudring A-V (2011) Stability and growth behavior of transition metal nanoparticles in ionic liquids prepared by thermal evaporation: how stable are they really? Phys Chem Chem Phys 13:7136-7141. https://doi.org/10.1039/c0cp02623a

Song H, Chen X (2003) Large-scale synthesis of carbon-encapsulated iron carbide nanoparticles by co-carbonization of durene with ferrocene. Chem Phys Lett 374:400-404. https://doi.org/10.1016/S0009-2614(03)00773-5

Sahoo P, Kamal S, Kumar T, Sreedhar B, Singh A, Srivastava S (2009) Synthesis of silver nanoparticles using facile wet chemical route. DSJ 59:447-455. https://doi.org/10. $14429 / \mathrm{dsj} .59 .1545$

Salas G, Podgoršek A, Campbell PS, Santini CC, Pádua AAH, Costa Gomes MF, Philippot K, Chaudret B, Turmine M (2011) Ruthenium nanoparticles in ionic liquids: structural and stability effects of polar solutes. Phys Chem Chem Phys 13:13527-13536. https://doi.org/10.1039/ c1cp20623k 
Sudhan, A. L. S.; Ramachandran, K.; Solomon, A. B.; Jawahar, C. P. (2021) Research progress on performance enhancement of heat pipes: a review. J Therm Anal Calorim. https://doi.org/10.1007/s10973-021-10732-3

Torimoto T, Okazaki K-I, Kiyama T, Hirahara K, Tanaka N, Kuwabata S (2006) Sputter deposition onto ionic liquids: simple and clean synthesis of highly dispersed ultrafine metal nanoparticles. Appl Phys Lett 89:243117. https:// doi.org/10.1063/1.2404975

Veith G, Lupini A, Pennycook S, Ownby G, Dudney N (2005) Nanoparticles of gold on $\gamma$-Al2O3 produced by dc magnetron sputtering. J Catal 231:151-158. https://doi.org/10. 1016/j.jcat.2004.12.008

Vekariya RL (2017) A review of ionic liquids: applications towards catalytic organic transformations. J Mol Liq 227:44-60. https://doi.org/10.1016/j.molliq.2016.11.123

Weissleder R, Elizondo G, Wittenberg J, Rabito CA, Bengele HH, Josephson L (1990) Ultrasmall superparamagnetic iron oxide: characterization of a new class of contrast agents for MR imaging. Radiology 175:489-493. https:// doi.org/10.1148/radiology.175.2.2326474

Wasserscheid P, Keim W (2000) Ionic liquids-new "solutions" for transition metal catalysis. Angew Chem Int Ed 39:3772-3789. https://doi.org/10.1002/1521-3773(20001 103)39:21\%3c3772:AID-ANIE3772\%3e3.0.CO;2-5

Wen Y, Huang W, Wang B, Fan J, Gao Z, Yin L (2012) Synthesis of $\mathrm{Cu}$ nanoparticles for large-scale preparation.
Mater Sci Eng, B 177:619-624. https://doi.org/10.1016/j. mseb.2012.02.026

Wang X, Huang Z, Yao Y, Qiao H, Zhong G, Pei Y, Zheng C, Kline D, Xia Q, Lin Z et al (2020) Continuous $2000 \mathrm{~K}$ droplet-to-particle synthesis. Mater Today 35:106-114. https://doi.org/10.1016/j.mattod.2019.11.004

Xiao D, Yuan D, He H, Gao M (2013) Microwave assisted onestep green synthesis of fluorescent carbon nanoparticles from ionic liquids and their application as novel fluorescence probe for quercetin determination. J Lumin 140:120 125. https://doi.org/10.1016/j.jlumin.2013.02.032

Yang H, Zhang J, Kumar S, Zhang H, Yang R, Fang J, Zou S (2009) Monodisperse and highly active PtNi nanoparticles for O2 reduction. Electrochem Commun 11:2278-2281. https://doi.org/10.1016/j.elecom.2009.10.009

Zheng W, Liu X, Yan Z, Zhu L (2009) Ionic liquid-assisted synthesis of large-scale $\mathrm{TiO} 2$ nanoparticles with controllable phase by hydrolysis of TiCl4. ACS Nano 3:115-122. https://doi.org/10.1021/nn800713w

Zhang S, Zhang Y, Wang Y, Liu S, Deng Y (2012) Sonochemical formation of iron oxide nanoparticles in ionic liquids for magnetic liquid marble. Phys Chem Chem Phys 14:5132-5138. https://doi.org/10.1039/c2cp23675c

Publisher's note Springer Nature remains neutral with regard to jurisdictional claims in published maps and institutional affiliations. 